\title{
Reformas pendientes en la organización de la actividad sanitaria*
}

\author{
Anna García-Altés \\ Agència de Qualitat i Avaluació Sanitàries de Catalunya (AQuAS), \\ Departament de Salut, Generalitat de Catalunya \\ Centro de Investigación en Economía y Salud. Universidad Pompeu Fabra

\begin{abstract}
Vicente Ortún
Departamento de Economía y Empresa, Universidad Pompeu Fabra

Centro de Investigación en Economía y Salud, Universidad Pompeu Fabra
\end{abstract}

\section{Resumen}

Se esquematiza la reciente evolución de la organización sanitaria en España analizando, e ilustrando con ejemplos, la innovación sanitaria. El énfasis del artículo se sitúa en la gestión sanitaria, tanto en la medida de su calidad como en la consideración del impacto de esta calidad en los resultados en salud. Se abordan las limitaciones de la gestión pública, que tanto influyen en las características de la gestión sanitaria, y se recuerda el deterioro institucional relativo de España para plantear las medidas que han de permitir mejorar la calidad de su gobierno. Se concluye con dos escogidas grandes vías de mejora de la gestión sanitaria: la centrada en la financiación pública y las que persiguen hacer individualmente atractivo lo socialmente conveniente.

Palabras clave: política sanitaria, regulación, salud pública, innovación organizativa, buen gobierno, financiación pública, gestión sanitaria.

Clasificación JEL: I18, H19, H44, H51, O31.

\begin{abstract}
The recent evolution of the healthcare organization in Spain is outlined, analyzing and illustrating with examples, healthcare innovation. The emphasis of the article is placed on healthcare management, both in terms of its quality and in the consideration of the impact of this quality on health outcomes. The limitations of public management, which influence the characteristics of healthcare management, are addressed, and the relative institutional deterioration of Spain is recalled in order to propose the measures that will allow improving the quality of its government. It concludes with two chosen big ways of improvement of healthcare management: the one centered in the public financing and the ones that pursue to make individually attractive what is socially convenient.
\end{abstract}

Keywords: health policy, regulation, public health, organizational innovation, good government, public financing, health management.

JEL classification: I18, H19, H44, H51, O31.

* Los autores desean agradecer los comentarios de Rosa Urbanos. Vicente Ortún reconoce la financiación del Ministerio de Economía, Industria y Competitividad (Proyecto ECO2017-83771-C3-2-R, Encaje público-privado en sanidad, del Programa Estatal de I+D+i orientada a los retos de la sociedad). 


\section{Tipología organizaciones}

Pese a que precios y mercados coordinan las decisiones individuales, las organizaciones existen, como ya escribió Coase en 1937, porque las transacciones tienen costes y, si en el seno de una organización son más pequeños, la organización está justificada y sus límites establecidos: allí donde los costes de realizar una transacción adicional dentro de la empresa se igualen con los costes de efectuar transacciones con el exterior.

Este enfoque de costes de transacción se ha calificado de neohobessiano pues, de la misma manera que en Hobbes los ciudadanos sacrifican libertades individuales para que el Estado funcione, en la organización serían sus integrantes racionales los que sacrificarían libertades individuales y contratarían a un supervisor para que controlase su labor. Esto fue lo que hicieron, según Benito Arruñada (1994), las cooperativas de remeros del bajo Yang-Tse durante la Revolución Cultural: dado que, habiendo enviado a los gerentes a los campos para ser reeducados, los remeros no pegaban «palo al agua» y acababan en el mar de China; tuvieron que contratar un capataz. ;Oh Lord, give us a boss! En ambos casos, Estado o empresa, se produce el sacrificio de una libertad individual -de matar o escaquearse- por el interés colectivo.

Los costes de transacción, como se sabe, son los de hacer funcionar el sistema: descubrir con quién se quiere tratar, informar de la intención de negociar y en qué términos, negociar, acordar un contrato, vigilar el cumplimiento de los pactos, etc. Incluyen costes de coordinación -en los que hay que incurrir aunque los intereses de las partes coincidan- y costes de motivación. Estos costes de transacción aumentan en relación directa con las asimetrías informativas y los problemas de información, ambos muy prevalentes en sanidad.

Las organizaciones han de establecer cómo se reparten los derechos de decisión, cómo se evalúa el comportamiento y cómo se incentiva, algo que en mercados con derechos de propiedad garantizados se produce de manera espontánea. El trípode organizativo no resulta sencillo en sanidad, especialmente su segunda pata -la de evaluar comportamientos- y su tercera, la de incentivar. Evaluar comportamientos requiere saber medir tanto productos intermedios, normalmente con agrupaciones de pacientes tipo clinical risk groups, como productos finales -impacto en el bienestar-, temas que aún están lejos de resolverse. Tampoco es sencilla la tarea de incentivar la calidad por la posibilidad de seleccionar casos que tienen los profesionales. Asuntos complejos que, ciñéndonos al mundo de las organizaciones, pasan por valorar las ventajas e inconvenientes del trabajo por cuenta ajena o propia, pero que no pueden desvincularse ni de las «reglas sociales de juego» (normas clínicas, influencia de la industria, etc.) ni de los incentivos individuales o de grupo (promoción, prestigio, estabilidad, reconocimiento, salario, etc.).

Según quien se apropie de las rentas residuales (beneficios) y según quien sea el titular de los derechos residuales de decisión (los no contemplados por la ley, el contrato o la costumbre), puede establecerse una tipología de las formas organizativas entre las puramente privadas y las puramente públicas (Cuadro 1). 


\section{CUADRO 1}

TIPOLOGÍA DE FORMAS ORGANIZATIVAS

\begin{tabular}{|c|c|c|c|c|}
\hline \multirow{5}{*}{ 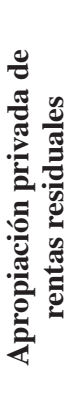 } & & \multicolumn{3}{|c|}{ Titular derechos de decisión } \\
\hline & & Privada & Mixta & Pública \\
\hline & Sí & $\begin{array}{l}\text { Privada pura } \\
\text { (capitalista) }\end{array}$ & Privada regulada & $\mathrm{X}$ \\
\hline & Parcialmente & $\begin{array}{l}\text { Comercial no } \\
\text { lucrativa }\end{array}$ & $\begin{array}{l}\text { Comercial lucrativa } \\
\text { con participación } \\
\text { pública }\end{array}$ & Pública comercial \\
\hline & No & No lucrativa pura & $\begin{array}{l}\text { Fundación privada } \\
\text { con financiación } \\
\text { pública }\end{array}$ & Pública pura \\
\hline
\end{tabular}

FUENTE: Elaboración propia.

Observamos en el Cuadro 1 diversas formas intermedias entre la organización pública pura y la organización privada pura que se pueden adaptar a las particularidades de la actividad sanitaria habida cuenta tanto de sus fallos del mercado como de sus fallos del Estado. En el mundo desarrollado prevalecen las organizaciones privadas no lucrativas y en España las públicas puras.

La Economía de las Organizaciones, con varios Nobeles en su haber -los últimos, en 2016, a Hart y Holmström- ayuda a pensar sobre las mejores formas de asignar derechos de decisión, evaluar comportamientos e incentivar, pero no produce recetas inmediatas sobre una cuestión tan contingente como la mejor organización sanitaria de un país, que depende tanto de sus valores y calidad institucional como de las virtudes y defectos del Estado. Por ello, en este artículo el énfasis se sitúa en la gestión. Se esquematiza, en el segundo epígrafe, la evolución organizativa y se analiza con mayor detalle y concreción la innovación organizativa ${ }^{1}$. El tercer epígrafe se dedica al empeño, recientemente conseguido, de medir la calidad de la gestión, conocer los determinantes de la misma y tratar de establecer el impacto de la calidad de la gestión en los resultados en salud. El cuarto epígrafe se centra en la gestión pública; tras establecer sus limitaciones se recuerda el deterioro institucional relativo de España y se plantean medidas para mejorar la calidad de su gobierno, en un análisis que debe mucho a otro artículo de este número (Hernández-Aguado, 2018). Se concluye, en un quinto epígrafe, con dos escogidas grandes vías de mejora de la gestión sanitaria: la centrada en la financiación pública y las que persiguen hacer individualmente atractivo lo socialmente conveniente.

\section{Evolución e innovación organizativa}

En la sanidad española coexiste la gestión directa de servicios -con una muy ligera incorporación de nuevas formas de gestión como consorcios, fundaciones,

\footnotetext{
${ }^{1}$ Las fórmulas de colaboración público-privada han centrado, posiblemente de forma muy desproporcionada, la atención en España y son objeto de análisis en otro artículo de este número.
} 
o sociedades mercantiles públicas- con la gestión indirecta de servicios mediante, sobre todo, conciertos y, muy secundariamente, concesiones de obra y concesiones administrativas, mecanismos de compra pública innovadora y contratos de riesgo compartido. En general, puede afirmarse que no se ha cogido por los cuernos el toro de la reforma de la administración pública, que tanto afecta a sanidad, a la que más adelante nos referiremos. Dejando atrás las cesantías decimonónicas cabe destacar:

- El Estatuto Maura de 1918 que introduce la inamovilidad y las oposiciones.

- La Ley de Funcionarios Civiles del Estado de 1964.

- La Ley 30/1984 de Medidas para la Reforma de la Función Pública.

- La Ley 55/2002, Estatuto Marco del personal del sistema nacional de salud.

- La Ley 7/2007, Estatuto Básico del Empleado Público.

- Real Decreto Legislativo 5/2015 por el que se aprueba el texto refundido de la Ley del Estatuto Básico del Empleado Público.

- La Ley 15/2014 de racionalización del sector público y otras medidas de reforma administrativa, bastante vacuas y poco comparables con la reforma de la Administración Pública, 1956-1958, por su alcance transformador (López Rodó, 1958).

Como dice José-Ramón Repullo², el estatuto marco del Sistema Nacional de Salud define casi todos los aspectos relevantes de la política de personal: acceso al puesto, retribución, negociación de las condiciones de trabajo, representación, etc. Esta rigidez se adapta mal a la misión y necesidades de los servicios sanitarios modernos, y lleva paradójicamente a un alto nivel de empleo inestable y abuso generalizado de contratos temporales.

Se conoce por innovación la generación de un bien o servicio de valor comercial o, en ausencia de mercado, apreciado por las personas. El sector salud, especialmente intensivo en conocimiento, alta especialización e intensidad tecnológica, es también altamente innovador. En este sector, la innovación busca mejorar los resultados de salud de la comunidad social y satisfacer las necesidades asistenciales personalizadas de los usuarios. Sería importante compartir una métrica común para medir la innovación pues, entre otras cosas, serviría para orientar la innovación hacia donde interesa. Parecería claro que en el sector sanitario esa métrica la pueden proporcionar la cantidad y calidad de vida resumida en los años de vida ajustados por calidad (AVAC). No obstante, y hasta ahora, han fracasado los intentos de que, en Europa, se adopten los AVAC como métrica compartida pese a la existencia de una directiva que busca una cierta homogeneización de la evaluación de tecnologías sanitarias entre los países de la Unión Europea.

La introducción de nuevos fármacos y productos sanitarios, junto con las innovaciones y la implementación de las tecnologías de la información y comunicación, son los tipos más reconocibles de innovación. Muchas innovaciones organizativas

\footnotetext{
${ }^{2}$ https://www.diariomedico.com/profesion/un-estatuto-para-el-medico-mas-flexible.html.\#
} 
surgen en el entorno de los centros sanitarios y suponen el traspaso del ámbito en que se da la asistencia (hospitalaria, ambulatoria o domiciliaria), implican la creación de nuevas unidades o la ampliación de los usos o funciones de unidades existentes, el establecimiento de acuerdos y circuitos con otros centros asistenciales para tratar determinadas enfermedades, o la integración en redes de cooperación con finalidades asistenciales o investigadoras. Las iniciativas legislativas de mayor alcance también entrarían dentro de las innovaciones organizativas.

Entre las diferentes maneras de caracterizar la innovación conviene destacar:

- Innovación en producto -o servicio-, en proceso, u organizativa. En el sector sanitario, la innovación en producto es la más frecuente (el robot quirúrgico, la historia clínica electrónica, las estatinas, la mamografía o el TAC), más que la innovación en proceso (los listados de comprobación quirúrgicos, la cirugía mayor ambulatoria, la cirugía laparoscópica) o la innovación organizativa (los códigos de actuación rápida, la integración de servicios sanitarios y sociales, hospitalización a domicilio, concentración de la cirugía de alta especialización). La innovación en producto atrae tanto a profesionales como a usuarios, y su introducción -como en el caso de los robots quirúrgicos- se realiza con un alcance no justificado por su impacto en el bienestar. En cambio, la innovación organizativa suele ser el patito feo y no únicamente en España. Existen ejemplos mundiales -Kaiser Permanente, Geiseinger, Mayo, Veterans Administration en EE. UU. o Aravind de Madurai y Narayana Hrudayalaya en Bangalore, ambos en India- de innovaciones organizativas que no se difunden como se difundirían en un sector más sometido a competencia o con una regulación menos restrictiva de la misma. Y las oportunidades para explotar economías de escala, gama y aprendizaje, con criterios de capacidad resolutiva, continúan existiendo en el sector sanitario.

- Efecto sustitución o expansión. Frecuentemente la extensión de las innovaciones médicas a lo largo del tiempo produce simultáneamente una reducción de sus costes unitarios y un aumento de los costes totales. La explicación de esta aparente paradoja reside en los efectos conocidos como de sustitución y expansión. El efecto sustitución se produce cuando una técnica de diferente intensidad -sea en dotación tecnológica o en consumo de recursos- reemplaza a una previa. El efecto expansión consiste en la prestación a pacientes con menor gravedad o síntomas más leves de tratamientos que previamente no se les habrían administrado, o en la prestación de tratamientos más intensivos.

- Valor de la innovación. Desde una perspectiva amplia, la innovación sanitaria ha proporcionado unos beneficios en salud que valen más que los notables costes que ha supuesto. Desde los conocidos análisis históricos de Fogel (1994) o McKeown (1976) en los que se pone de manifiesto el papel secundario de la atención sanitaria en la mejora de la salud de las poblaciones -en comparación con las mejoras en la nutrición, la higiene y el manejo de la reproducción-, hasta los análisis de Cutler et al. (2001), que compara cómo han cambiado 
precios, cantidades e intensidad en los procesos (otitis media, infarto agudo de miocardio o depresión mayor) con los resultados en términos de cantidad y calidad de vida de esos procesos. Ni el enfoque de análisis coste-beneficio generalizado, a la Cutler, ni siquiera el análisis coste-beneficio, ha de esperarse que sean de uso cotidiano por la resistencia a valorar monetariamente las ganancias en salud. Cabe, no obstante, con el mucho más extendido empleo del análisis coste-efectividad, tratar de llegar a una métrica común para medir el valor de la innovación: La cantidad y calidad de vida medida, como se ha dicho ya, en años de vida ajustados por calidad (AVAC).

- Innovación con base científica o sin ella. Ni Inditex, ni el Cirque du Soleil, ni Ikea, ni el container o la palette han resultado de la investigación. Y no por ello dejan de ser innovaciones.

- Grado de disrupción de la innovación. Como insistiremos más adelante, la mayoría de las empresas disruptivas ingresan a un mercado con un producto cuyo valor es menor que el de los operadores tradicionales del mercado, pero su costo es mucho menor. Ese es el modelo para los disruptores clásicos, como Southwest Airlines, MP3 o los fabricantes japoneses de automóviles. El cuidado de la salud tiende a ser diferente, porque los consumidores generalmente no quieren conformarse con un producto de menor calidad, incluso si es sustancialmente más barato.

- Velocidad de la difusión. Un aspecto crucial de la innovación es su velocidad de difusión. Entre la lentitud de la difusión de los cítricos para la prevención del escorbuto y la fulgurante difusión del éter como anestésico, la historia ha conocido múltiples velocidades intermedias como la del ácido carbólico de Líster para evitar las sepsis tras las intervenciones quirúrgicas. En épocas más recientes la colaboración Cochrane vino motivada por el lapso habido entre la demostración de la eficacia del tratamiento del infarto de miocardio con trombolíticos en los años setenta y su uso generalizado en los años noventa.

En el libro Innovaciones en gestión clínica y sanitaria (2005), Ricard Meneu, Vicente Ortún y Fernando Rodríguez Artalejo hacen un repaso a las innovaciones más relevantes entre 1980 y 2000, de las que hacen un análisis pormenorizado de nueve de ellas: actuaciones contra el tabaquismo, la evaluación de medicamentos sobre bases más científicas, atención primaria de salud, los sistemas de ajuste de riesgos, programa MIR, extracción de cataratas e implantación de lentes intraoculares, los programas de gestión de enfermedades, los medicamentos cardiovasculares y PubMed.

Sin voluntad de exhaustividad, aquí se describen brevemente algunas innovaciones organizativas más recientes que han tenido buenos resultados en la salud de la población:

- La atención a determinadas patologías en torno a «códigos»: son códigos de emergencia que llevan a la activación inmediata de una red de servicios de salud 
dirigidos a proporcionar una atención urgente y adecuada para los pacientes con patologías tiempo-dependientes. La necesidad de actuar con rapidez ha llevado a varias comunidades autónomas a territorializar la atención, involucrando a los hospitales de la comunidad y de referencia, sus zonas de captación y los sistemas de emergencias médicas. Este es el caso del ictus agudo, el infarto agudo de miocardio y el paciente politraumático. En Cataluña, y para el caso del ictus agudo, se ha estimado que, en el periodo 2006-2012, la existencia del código ha permitido evitar 719 defunciones que se habrían producido si se hubieran mantenido las tasas de incidencia y supervivencia existentes (García-Altés et al., 2016).

- Teledermatología (extensible a otras especialidades: el médico de familia ve la lesión de la piel, la fotografía y la adjunta con el seguimiento clínico a la historia clínica electrónica. En el mismo momento, se añade al paciente en la agenda de teledermatología y en la agenda de su médico de familia una semana más tarde. El profesional dermatólogo usa esta agenda para revisar las fotografías y escribir el diagnóstico y la pauta de tratamiento y seguimiento. Una semana después, el médico de familia revisa el caso y llama al paciente con los resultados. Si el dermatólogo lo cree necesario, cita al paciente para una visita presencial. La implantación de la teledermatología en la comarca del Bages ha demostrado ser una buena estrategia para conseguir reducir la lista de espera (Vidal-Alaball et al., 2015).

- Preoperatorio online: consiste en una visita virtual donde el profesional anestesista recoge la información relevante de la historia clínica y las pruebas complementarias de la atención primaria y hospitalaria. Después de que un profesional médico o enfermero revise la información, el profesional enfermero contacta con el paciente para confirmar la información y gestionar la programación de las pruebas pendientes y posterior visita. La experiencia del Hospital de Viladecans ha estimado un ahorro de 25 euros por proceso programado solo teniendo en cuenta el ahorro en pruebas complementarias ${ }^{3}$.

- Fast-track en cirugía colorrectal (como ejemplo) es una nueva estrategia para intentar mejorar la evolución postoperatoria de los pacientes intervenidos de colon o recto, con el objetivo de disminuir el sufrimiento de los enfermos, que incluye las siguientes medidas: informar al paciente del proceso global, incluida la previsión del alta, y comprometerlo en las decisiones, promover medidas de preservación de la función intestinal, evitar las medicaciones que laxen los intestinos, preservar la temperatura corporal durante la intervención, evitar los sondajes, ajustar la hidroterapia, anestesiar por vía peridural, usar técnicas quirúrgicas de invasión mínima, evitar los analgésicos opiáceos, hacer prevención de náuseas y vómitos, retirar catéteres lo antes posible o iniciar la alimentación oral y la deambulación de manera precoz. Los estudios existentes en nuestro entorno muestran una disminución de la estancia media de entre tres y seis días (Gil Egea et al., 2008 y Esteban et al., 2012). 
- Enfermera gestora de la demanda. Se define como la respuesta que da la enfermera a un paciente que presenta un problema de salud y que necesita una resolución preferente. Se diferencia del triaje porque, mientras que la gestión de la demanda pretende dar una solución al problema de salud planteado, la selección se refiere a la recepción, acogida y clasificación de pacientes que llevan a cabo las enfermeras en los servicios de urgencias. La gestión enfermera de la demanda ha demostrado presentar una alta resolución de las afecciones atendidas, aportando a la vez un porcentaje elevado de consejos de salud y de educación sanitaria en las visitas atendidas (Brugués et al., 2016). España tiene una ratio de médicas/enfermeros muy desequilibrada en contra de la enfermería, extraordinariamente competente en ámbitos como el de cuidados intensivos, lo cual dificulta aplicar el sentido común para organizar la atención según el principio de capacidad resolutiva, que exige que cada problema sea atendido por quien consigue la mayor efectividad para unos costes determinados.

- Concentración de la cirugía oncológica digestiva de alta especialización. La concentración de la cirugía oncológica digestiva de alta especialización se inició en Cataluña a principios del año 2012, consolidándose en 2015. La comparación entre los periodos previo (2005-2011) y posterior (2012-2015) a la concentración, arroja como resultado que la mortalidad a los 30 días mejoró de manera significativa con reducciones del 79 por 100,41 por 100 y 50 por 100 en cirugía de cáncer de esófago, de páncreas y de metástasis hepáticas, respectivamente (Manchón-Walsh et al., 2017).

- «Ley antitabaco». La Ley 28/2005 hizo obligatoria la incorporación de advertencias sanitarias en el etiquetado del tabaco, prohibió su publicidad y promoción e implantó la regulación parcial de su consumo en los lugares públicos (Ley 28/2005). Posteriormente la Ley 42/2010 introdujo la restricción total del consumo de tabaco en lugares públicos cerrados, lo que provocó una caída brusca del 9,41 por 100 en las ventas de cigarrillos (Pinilla et al., 2017), sin que disminuyera la actividad en bares y restaurantes (García-Altés et al., 2015).

- Medidas sobre seguridad vial, entre las que destaca el sistema de pérdida de puntos por infracciones de tráfico con sanción solo administrativa (Ley 17/2005) y la tipificación penal de todas las conductas de conducción temeraria (Ley 15/2007). Su evaluación muestra una reducción significativa en el riesgo de tener un accidente en ambos sexos, todas las edades y todos los medios de transporte, excepto para los peatones (Novoa et al., 2010a; Novoa et al., 2010b; Novoa et al., 2011). Tanto la actuación sobre el tabaquismo como la realizada sobre la seguridad vial, constituyen las dos medidas de política que más han contribuido a la ganancia de años de vida en España en épocas recientes, con un importante ahorro de costes para la sociedad (García-Altés et al., 2013).

${ }^{4}$ Al estilo del antiguo Canalejo de A Coruña, en el que el recordado Alfonso Castro Beiras fue jefe de Servicio de Cardiología. 
Para terminar, destacamos aquí algunas innovaciones que, sin ser muy recientes, ofrecen resultados satisfactorios, como la organización de hospitales por áreas ${ }^{4}$, el desempeño «por cuenta propia» de las entidades de base asociativa en atención primaria, la atención multidisciplinar en cáncer y numerosas iniciativas que, aunque aparentemente funcionan, no llegan a ser formalmente evaluadas.

\section{Medida de la calidad de la gestión y sus determinantes}

\subsection{La gestión como profesión}

Hasta 1860-1880 los mercados eran fundamentalmente locales y el tamaño de las empresas reducido. Tres desarrollos tecnológicos -barcos a vapor, ferrocarriles y telégrafo- alteran el panorama, pues facilitan la producción en masa e implican cambios en las organizaciones: nuevos sistemas de información y contratación de gerentes.

A partir de 1880, con la Segunda Revolución Industrial, se transforman las industrias antiguas (acero, cobre, aluminio, refinamiento de aceite, de azúcar, envasado y embotellado, productos agrícolas, etc.) y se crean nuevas industrias: químicas (tinturas, medicinas, fibras, abonos, etc.), maquinaria agrícola, de oficina y doméstica (máquinas coser, por ejemplo), y de generación y transmisión de electricidad (alumbrado, tracción urbana, poder industrial, etc.).

Las nuevas empresas, tanto en los sectores tradicionales como en los nuevos sectores, diferían de las antiguas en que eran mucho más intensivas en capital y capaces de explotar las economías de escala y gama que las nuevas tecnologías permitían.

La gestión como profesión empieza a aparecer en esas grandes organizaciones cuando surge como función especializada diferente a la propiedad. Gestión, en aquel contexto post Segunda Revolución Industrial, suponía precisamente explotar esas potenciales economías de escala y gama: $a$ ) se requería gran coordinación para mantener una producción elevada, así como un flujo ininterrumpido de materias primas y productos acabados, y $b$ ) hacían falta unos conocimientos y habilidades para conseguir las citadas economías de escala y gama. Las empresas que lo consiguieron han seguido dominando sus sectores durante todas las décadas transcurridas. Sea Exxon-Mobil (descendiente de Rockefeller Standard Oil y su espectacular concentración del refinado de keroseno) o Bayer, Basf y Hoechst (ahora integrada en Sanofi-Aventis), que en el valle del Rin consiguieron grandes economías de gama entre tintes y fármacos (Chandler, 1990). Han sido las empresas capital-intensivas que explotaron economías de escala y de gama invirtiendo en producción, distribución y gestión, las que dominaron sus industrias rápidamente (Tafunell y Carreras, 2010). Y muchas continuaron haciéndolo por décadas; en la industria farmacéutica tenemos buen ejemplo de ello. 


\subsection{La gestión como disciplina: entre «Fosbury» y los «antígenos»}

Las profesiones tradicionales, tipo Medicina y Abogacía, tienen claramente definidos los conocimientos, habilidades y aptitudes deseables para su ejercicio, siendo éstos, además, evaluables por colegas. No existe una claridad equiparable entre profesionales gestores, seleccionados con frecuencia a través de cazatalentos, tal vez tanto por la debilidad de su base disciplinaria como por la mezcla indiscriminada de «Fosbury» (hacer las cosas como quien mejor las haga) y «antígenos» (doctrinas extrañas que disparan una respuesta inmunitaria que bloquea la curiosidad por el abordaje científico de la gestión).

Respecto la base disciplinaria resultó ilustrativo el debate que se produjo hace 25 años entre la obra de John Kay (1993), The foundations of corporate success, y la de Paul Milgrom y John Roberts (1992), Economics, Organization and Management. El primero pretendía responder al título de «fundamentos del éxito empresarial» tratando de establecer afirmaciones universales y contrastables sobre los factores determinantes del éxito empresarial. Los segundos pretendían simplemente facilitar conceptos y análisis que ayudaran a pensar sobre los problemas de gestión. El libro de Milgrom y Roberts se acabó convirtiendo en el mejor texto de Economía de las Organizaciones y, en cambio, el de Kay no ha sido su obra más destacada. El hecho de que «cómo gestionar para tener éxito» no sea identificable con una disciplina científica única no impide que pueda hablarse de ciencias de la gestión. ¿Cómo? Si gestionar supone coordinar y motivar a los miembros de la organización para conseguir los objetivos de ésta, cualquier disciplina, Psicología o Logística, que realice afirmaciones universales y contrastables sobre cómo motivar o coordinar mejor constituirá indudablemente una ciencia de la gestión.

Más fácil ha resultado ir discriminando entre los que denominamos componentes «Fosbury» de la gestión de los componentes «antígeno». Entre los sorprendentes récords que se produjeron en los Juegos Olímpicos de México en 1968 destaca el de Fosbury, pues fue motivado por un cambio en la técnica en salto de altura: de rodillo a espalda. Desde entonces, ningún atleta que pretenda clasificarse puede saltar de otra manera. Únicamente hay una forma de hacer las cosas: como quien las hace mejor. Algo parecido sucede con las empresas si sustituimos el clasificarse para unas Olimpíadas por ser capaz de producir y vender en mercados nacionales e internacionales. A lo largo del siglo pasado, fueron técnicas «Fosbury» destacadas la organización científica del trabajo de Taylor, los estudios de Hawthorne (Shewhart) en la década de los treinta, que dieron origen tanto a la psicología industrial como a lo que mucho tiempo después se conoce como «calidad total», los avances en producción y logística habidos durante la Segunda Guerra Mundial (McNamara), el toyotismo, etc.

\footnotetext{
5 Según Paul Krugman, la literatura económica puede clasificarse en tres grandes familias: la escrita parcialmente en griego, la del sube y baja y la de aeropuerto. Esta última suele ser entretenida, raramente bien informada y nunca seria. https://www.nytimes.com/1990/10/28/books/books-business-economics-101.html.
} 
Tampoco resulta difícil identificar la literatura «antigénica»: aquella que genera anticuerpos persistentes en el tiempo a cualquier texto de gestión. La exposición puede producirse fácilmente en aeropuertos ${ }^{5}$ y son más proclives a la reacción inmune las personas formadas con cierta capacidad de razonamiento lógico y familiarizadas con la literatura científica; exactamente el tipo de personas que suele darse entre los profesionales sanitarios.

Tanto el «Fosbury» como los «antígenos» han sido el resultado de la actividad de académicos (de Simon a Kahneman), gurús como Drucker, académicos con toque gurú como Porter, practicones con éxito que suelen olvidar el papel que la suerte juega (Sloan, Welch), y satíricos (Parkinson o los Lynn-Jay, de «Sí, Ministro»). Entre los académicos empiezan a tener una contribución importante los economistas más interesados por el comportamiento y la experimentación.

\subsection{La medida de la calidad de la gestión}

Ha tenido que llegar el siglo XXI para disponer de una medida validada de la calidad de la gestión, la accesible en el World Management Survey. Cualquier empresa manufacturera, hospital, distribuidor o escuela puede además compararse con los demás introduciendo sus datos.

Bloom, Genakos, Sadun y Van Reenen (2012) han sido sus artífices: encuestaron a más de 12.000 empresas de diversas industrias en 34 países para recopilar información sobre la calidad de las prácticas de gestión dentro de cada empresa. Usando estas respuestas de la encuesta, construyeron una medida de cómo se gestionaba una empresa, que iba desde 1 (peor práctica de gestión) a 5 (mejor práctica de gestión). Esta medida les permitió comparar las prácticas de gestión entre industrias y países.

La herramienta de evaluación midió las prácticas de gestión en cuatro áreas clave. Primera, gestión de operaciones: ¿se utilizan técnicas de producción y distribución ajustadas (las lean originarias de Toyota)? Segunda, monitoreo: ¿cómo las organizaciones monitorean lo que sucede dentro de la empresa y cómo utilizan esta información para la mejora continua? En tercer lugar, los objetivos: ¿las organizaciones establecen los objetivos correctos?, ¿consiguen los resultados correctos y toman las medidas adecuadas si los dos son inconsistentes? En cuarto lugar, los incentivos: ¿las organizaciones promueven y recompensan empleados basados en el desempeño, priorizando la contratación y tratando de mantener su mejor desempeño?

Se utilizó un mecanismo de doble ciego. Esencialmente ni los entrevistadores sabían qué «examinaban» ni los entrevistados conocían que eran «examinados». Con posterioridad a la validación del instrumento de encuesta, tanto un experimento natural como otro diseñado respaldaron la validez del instrumento. La crisis del 2008 afectó menos a las empresas con «buena» gestión (experimento natural) y las empresas textiles de la India -escogidas aleatoriamente- adiestradas sobre gestión mejoraron en un año su productividad un 17 por 100 más que las del grupo control (experimento diseñado). 


\subsection{La gestión en el sector sanitario}

La gestión en el sector sanitario presenta tres ámbitos diferentes: el de la intervención del Estado -regulando, financiando, informando, produciendo incluso- que se traduce en política sanitaria, el de la gestión de centros y el de la gestión clínica (Figura 1).

Como en otros sectores, los beneficios pueden resultar socialmente beneficiosos si premian la eficiencia, la innovación y compensan el riesgo, y socialmente perjudiciales si se obtienen en base a restringir la competencia, el aprovechamiento de contactos y la captura regulatoria (la riqueza crea poder, el poder crea riqueza). Más que en otros sectores, el incentivo del beneficio tiene gran capacidad para cortocircuitar la búsqueda de mejoras en la cantidad y calidad de vida de pacientes y poblaciones. La Economía de la Salud se esfuerza en compatibilizar ambos objetivos (pagos capitativos, por ejemplo), pero se reconoce, tanto la mayor seguridad que proporcionan los incentivos débiles al frenar impulsos difícilmente refrenables, en usuarios y en profesionales, a «hacer más» al margen de su indicación, como las dificultades de coordinación.

El grado de competencia constituye un factor exógeno que afecta a la calidad de la gestión. El sector sanitario está más «a prueba de Amazon» que otros sectores. De hecho, Amazon fracasó en su primera entrada al sector sanitario, como también lo hicieron Google o Microsoft, y su segunda entrada -gestionar la atención sanitaria de 1.200.000 empleados, los propios junto con la de los de Berskshire and J.P. Morgan en EE. UU.- es todavía muy reciente ${ }^{6}$. Conviene insistir en algo ya señalado con

FIGURA 1

\section{LOS TRES ÁMBITOS DE LA GESTIÓN SANITARIA}

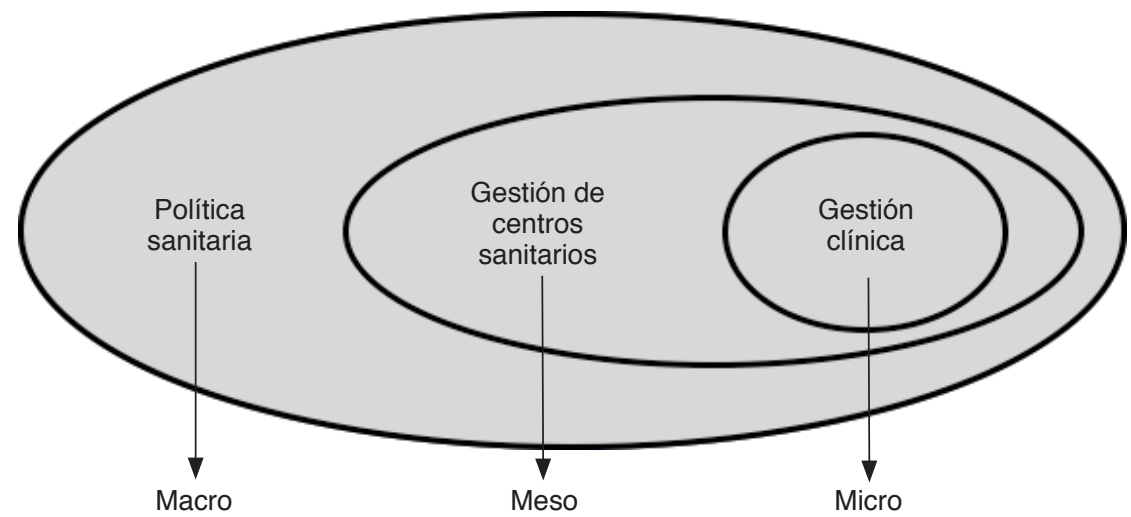

FUENTE: ORTÚN, V. (1993). «Presentación del V Congreso de la Sociedad Española de Salud Pública y Administración Sanitaria». Gaceta Sanitaria, 7 (38S), 1-2.

${ }^{6}$ MARGOT SANGER-KATZ y REED ABELSON. «Can Amazon and friends handle heath care? There's reason for doubt». New York Times, 30 enero 2018. 
anterioridad: que la mayoría de las empresas disruptivas ingresan a un mercado con un producto cuyo valor es menor que el de los operadores tradicionales del mercado, pero su costo es mucho menor. Ese es el modelo para los disruptores clásicos, como Southwest Airlines, MP3 o los fabricantes japoneses de automóviles. El cuidado de la salud tiende a ser diferente, porque los consumidores generalmente no quieren conformarse con un producto de menor calidad, incluso si es sustancialmente más barato.

La calidad de los hospitales mejora cuando compiten por comparación en calidad (Bloom et al., 2015). Se aplicó la ya mencionada encuesta de valoración de la calidad de la gestión, validada en otros sectores de la economía y en otros países, a dos tercios de los hospitales de agudos de Inglaterra. Se agrupó un total de 18 prácticas en cuatro dimensiones: a) Operaciones ajustadas (lean), como admisión, protocolización, interconsultas, alta y seguimiento, etc.; b) Medida de la actuación en base a la adopción de tecnología, la prevención de errores, la mejora continua, etc.; c) Fijación de objetivos; d) Incentivos: atracción y retención de talento, criba de incumplidores, etc. Resultó que las mejores prácticas gestoras estaban asociadas con mejores resultados, incluyendo menor mortalidad tras infarto agudo de miocardio, mejores resultados financieros, mayor satisfacción del personal y puntuaciones más altas por parte de la agencia supervisora de la calidad. La incorporación de una variable instrumental de tipo político permite establecer causalidad y su sentido: mayor competencia entre hospitales provoca una mejor calidad de la gestión. Aunque existan más formas de mejorar la calidad, como fomentar la elección informada por parte de los usuarios, los hallazgos del trabajo respaldan las políticas de los países que, como Holanda, Alemania, Reino Unido o Noruega, tratan de promover la competencia por comparación.

Sabemos que la garantía de inmortalidad a organizaciones y personas constituye receta infalible para el estancamiento y el embotamiento. La innovación nace en parte de la necesidad. Los derechos hay que garantizarlos, con las oportunas redes sociales de seguridad, pero los privilegios no.

Los servicios sanitarios no se importan, tampoco los beneficios extraordinarios permiten aproximar el grado de competencia y, para acabarlo de arreglar, muchos productores de servicios sanitarios son monopolios naturales. También la FIFA y la Agencia Tributaria, lo que no impide que fuera aconsejable mejorar los mecanismos de asignación de los campeonatos de fútbol, o ajustar los resultados de algunos colectivos profesionales (inspectores de hacienda, pero también profesores de universidad, clínicos, etc.). Y aunque las formas rígidas de organización, inadecuadamente regidas por el derecho administrativo, tenderán a flexibilizarse en los próximos treinta años, no será suficiente para estimular una mejor actuación si, allá donde se pueda, no se introduce una cierta competencia por comparación, lo que no requiere ni de mercados ni de profesión alguna de neoliberalismo.

No se trata ni de competir en precios (sacrificando las calidades que el usuario no percibe) ni de realizar experimentos a prueba de fallos, por el interés del promotor político en que luzcan bien, sino de ir introduciendo la idea de que los recursos que 
una organización sanitaria reciba dependerán, de entrada en una mínima parte, de la calidad que ofrezca en relación con sus comparables. La mayor integración, aunque sea virtual, que se precisa para atender a los polipatológicos con limitaciones en las actividades de la vida diaria, disminuirá el número de proveedores sanitarios y la mayor concentración resultante obligará, en ocasiones, a buscar comparadores más lejanos.

Los factores que influyen en la mejora de los resultados clínicos, financieros y la satisfacción del personal, además de la competencia por comparación en calidad, son el tamaño y las habilidades clínicas y gestoras. La asociación entre tamaño (volumen de intervenciones quirúrgicas, procedimientos, etc.) y calidad está bien establecida. Constituye la expresión clínica de las economías de escala y refleja la obviedad de que la maestría en un oficio se adquiere con la práctica, aunque no puede descartarse todavía que la derivación selectiva juegue asimismo un papel. Las habilidades clínicas y gestoras (mejor comunicación, mayor credibilidad y autoridad) -por este orden- explican un mejor comportamiento de las organizaciones sanitarias.

\subsection{Impacto de la gestión en los resultados en salud}

Aunque sea únicamente partiendo de estudios británicos y estadounidenses, algo puede decirse del impacto de la gestión -esa que ahora sabe medirse- en los resultados en salud, que hace tiempo vienen midiéndose. De entrada, los Chief Executive Officers (CEO) no parecen mejorar el rendimiento de los hospitales ingleses (Janke et al., 2018). Tal vez no tratan tanto de mejorar la eficiencia a medio-largo plazo como de evitar los impactos negativos en la opinión pública que más pudieran preocupar a quienes les han nombrado. Conviene recordar la tremenda inercia profesional y organizativa de los hospitales públicos que difícilmente alterará un CEO «interino».

Por otra parte, los hospitales con las mejores prácticas de gestión ofrecen mayor calidad. En hospitales con órganos de gobierno que miden los resultados clínicos, sus equipos de gestión presentan mejores resultados en gestión de personas, fijación de objetivos, seguimiento de resultados y gestión de operaciones (Tsai et al., 2015).

Se ha constatado también que la utilización de herramientas de gestión modernas (estandarización de procesos, medición de resultados, fijación de objetivos, gestión de incentivos) está correlacionada con una disminución de la mortalidad y la mejora del proceso asistencial en el infarto agudo de miocardio (McConnell et al., 2013 y 2016).

Se han evidenciado, finalmente, aspectos clave en las organizaciones como la comunicación eficaz, la colaboración entre profesionales, la participación del personal, la cultura de resolución de problemas y la innovación, y se han asociado estadísticamente con la disminución de las tasas de mortalidad por infarto agudo de miocardio (Bradley et al., 2012). Estas estrategias no requieren mucha inversión, pero sí nuevas formas de trabajo y organización. 


\section{Vías de avance en gestión pública}

La gestión pública, aunque substantivamente gestión, debe desenvolverse con unas limitaciones impuestas por la responsabilidad fiduciaria que constituye la contrapartida a la excepcionalidad del Estado como organización. En una organización privada, tanto la pertenencia a la organización como la distribución entre las partes de obligaciones y derechos resultan de arreglos contractuales (voluntarios en principio). En el Estado, en cambio, los ciudadanos pertenecen obligatoriamente a la organización, que tiene poderes de coacción ejercitables sin aquiescencia individual. Ello obliga al Estado a un comportamiento fiduciario que impone una serie de limitaciones, convenientes algunas, mitigables otras, bien por la investigación bien por la acción.

\subsection{Limitaciones a la gestión pública}

- Visibilidad y transparencia, que produce la reacción paradójica de secretismo en el proceso decisional, no siempre justificado por la relevancia estratégica del asunto abordado o por razones de respeto a la confidencialidad de los datos empleados.

- Control jurisdiccional constante.

- Alternancia de gobiernos, que hace más difícil contraer compromisos a largo plazo y que constituye un reflejo tanto de la heterogeneidad de preferencias de los principales (ciudadanos para los políticos, políticos para los organismos públicos, etc.) como de su inconsistencia temporal. En una empresa privada los propietarios exhiben preferencias consistentes y este problema no se presenta.

- Garantismo (más que legalismo), para impedir abusos de autoridad y vulneración de derechos individuales. La preocupación por la profilaxis de la arbitrariedad niega la discrecionalidad (o sea, la posibilidad de desplazarse por una isocuanta).

- Procedimentalismo burocrático que garantice la igualdad de trato (dado que no existe la opción «salida» para los individuos), con todas las ventajas e inconvenientes de la burocracia.

- Múltiples objetivos, impacto en el bienestar desconocido y rendimiento también difícil de apreciar por los problemas de definición y medida de la actividad de la administración.

- Dificultad de establecer mecanismos de competencia por comparación. Si, por ejemplo, solo existe una Agencia Tributaria, no cabe el patrón externo (benchmarking), pero sí mecanismos de torneo en el seno de la propia Agencia: léase, rendimiento de unidades comparables ajustado por las variables exógenas que las unidades no controlan (renta, tamaño poblacional, etc.).

- Captura por intereses de burócratas, políticos y lobbies. 
No todas estas limitaciones han de ser eliminadas, ya que muchas de ellas se derivan precisamente de las razones que llevan al Estado a intervenir: las de aumentar la equidad (pensiones con efectos redistributivos, subsidios familiares, seguros de desempleo, etc.) y las de corregir las disfunciones del mercado. Esta corrección de disfunciones implica: abordar las externalidades (educación básica, protección del medio ambiente, etc.), regular los monopolios, y corregir la información imperfecta (seguros de salud, reglamentación financiera, protección del consumidor) teniendo en cuenta cómo tales disfunciones quedan afectadas por el cambio tecnológico.

\subsection{El Estado, la calidad de las instituciones, «lo público» en general, recuperados}

Los informes del Banco Mundial de 1996 y 1997 ayudaron a restaurar la importancia del Estado y corregir la trampa ideológica creada por una interpretación completamente errónea de la caída del muro de Berlín: los enormes fallos de la transición de las economías planificadas al mercado. Las economías de Europa Central y del Este revelaron que el correcto funcionamiento de los mercados es solo una condición necesaria pero no suficiente: sin un Estado eficaz, los países fracasan. La realidad y el trabajo académico han ayudado a que el enfoque institucional sea ampliamente aceptado hoy. En resumen, se puede decir que para que una sociedad se desarrolle, es necesario que sus instituciones hagan individualmente atractivo lo que sea socialmente conveniente.

La medida de calidad institucional que ocupa más espacio en los medios de comunicación social es la de Transparency International. En ella, España ha pasado de ocupar la posición vigésima en el mundo en año 2000 a la cuadragésimo segunda en 2017, un muy notable descenso en la percepción de corrupción en el sector público por parte de diversos organismos internacionales que intervienen en la elaboración del índice (Figura 2). Otras medidas como el Rule of Law Index o el Índice de Imparcialidad de la Universidad de Gotemburgo también devuelven una imagen de cierto deterioro institucional en España.

Muy posiblemente, los indicadores mejor validados, con una trayectoria temporal iniciada en 1996, sean los Worlwide Governance Indicators del Banco Mundial, que recogen seis dimensiones de calidad de gobierno: voz y rendición de cuentas; estabilidad política y ausencia de violencia; efectividad gubernativa; calidad regulatoria; imperio de la ley (Estado de derecho); y control de la corrupción. La Figura 3 muestra datos para España entre 1996 y 2016: los intervalos de confianza dificultan sentenciar deterioro salvo que se considere la mejora registrada en otros países. Como reza el refrán: «Camarón que se duerme, se lo lleva la corriente». 
FIGURA 2

ÍNDICE DE PERCEPCIÓN DE CORRUPCIÓN 2017

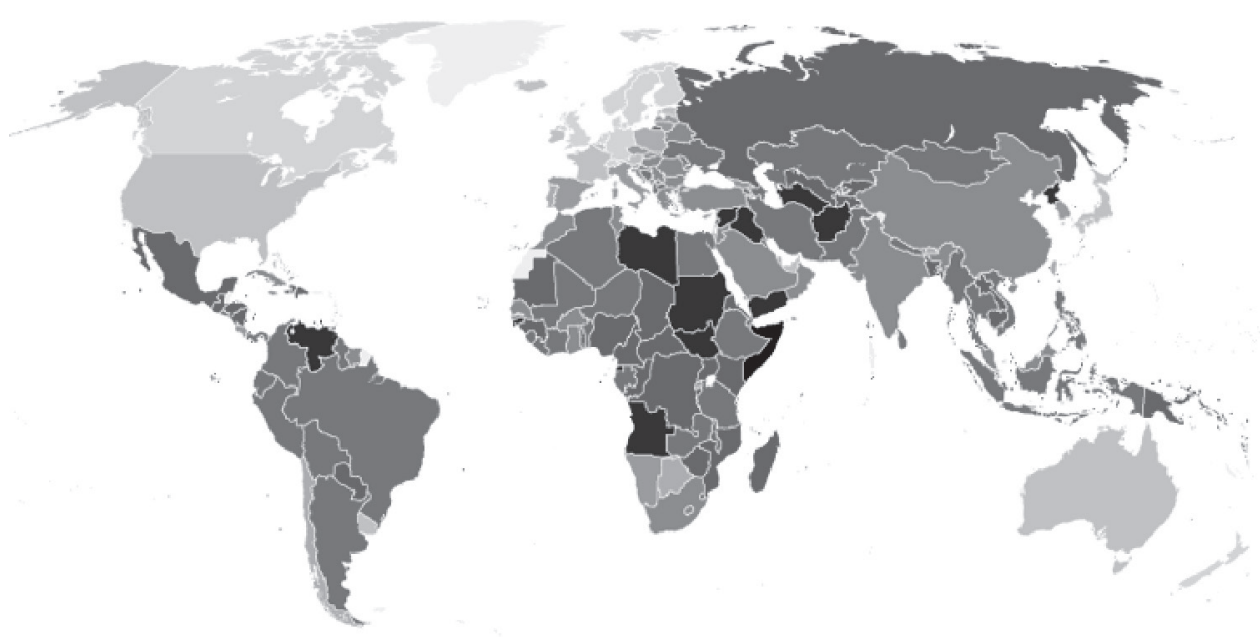

Puntuación

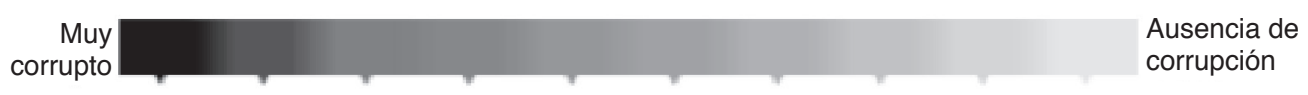

$\begin{array}{llllllllll}0-9 & 10-19 & 20-29 & 30-39 & 40-49 & 50-59 & 60-69 & 70-79 & 80-89 & 90-99\end{array}$

FUENTE: Transparency International.

\subsection{El deterioro institucional de España}

El deterioro institucional en España ha sido en parte un resultado inesperado de la Unión Monetaria de 1999 (el euro), que supuestamente traería ajustes estructurales y reformas institucionales a las economías menos competitivas, cuando ni la devaluación de la moneda ni el déficit público superior al 3 por 100 del producto interno bruto fueran ya permitidos. España ya ha incurrido en inversiones y gastos improductivos, ha sufrido la enfermedad holandesa ${ }^{7}$, la deuda está causando resaca y llevará un tiempo renovar las instituciones españolas (Fernández-Villaverde et al., 2013). El crecimiento español durante la expansión de 1994-2007 se basó en la acumulación de factores más que en las ganancias de productividad. En particular, el crecimiento anual de la productividad total de los factores fue del $-0,7$ por 100 , que es bajo en comparación con otras economías desarrolladas como las de la UE o

\footnotetext{
${ }^{7}$ En economía, la enfermedad holandesa es la aparente relación causal entre el aumento en el desarrollo económico de un sector específico (por ejemplo, la construcción o el turismo) y una disminución en otros sectores (como el sector manufacturero o la agricultura). A medida que los ingresos aumentan en el sector en crecimiento, la moneda se aprecia en comparación con las monedas de otras naciones. Esto hace que las otras exportaciones de la nación se vuelvan más caras y las importaciones más baratas, lo que hace que esos sectores sean menos competitivos.
} 
FIGURA 3

INDICADORES DE BUEN GOBIERNO: ESPAÑA, 1996-2016

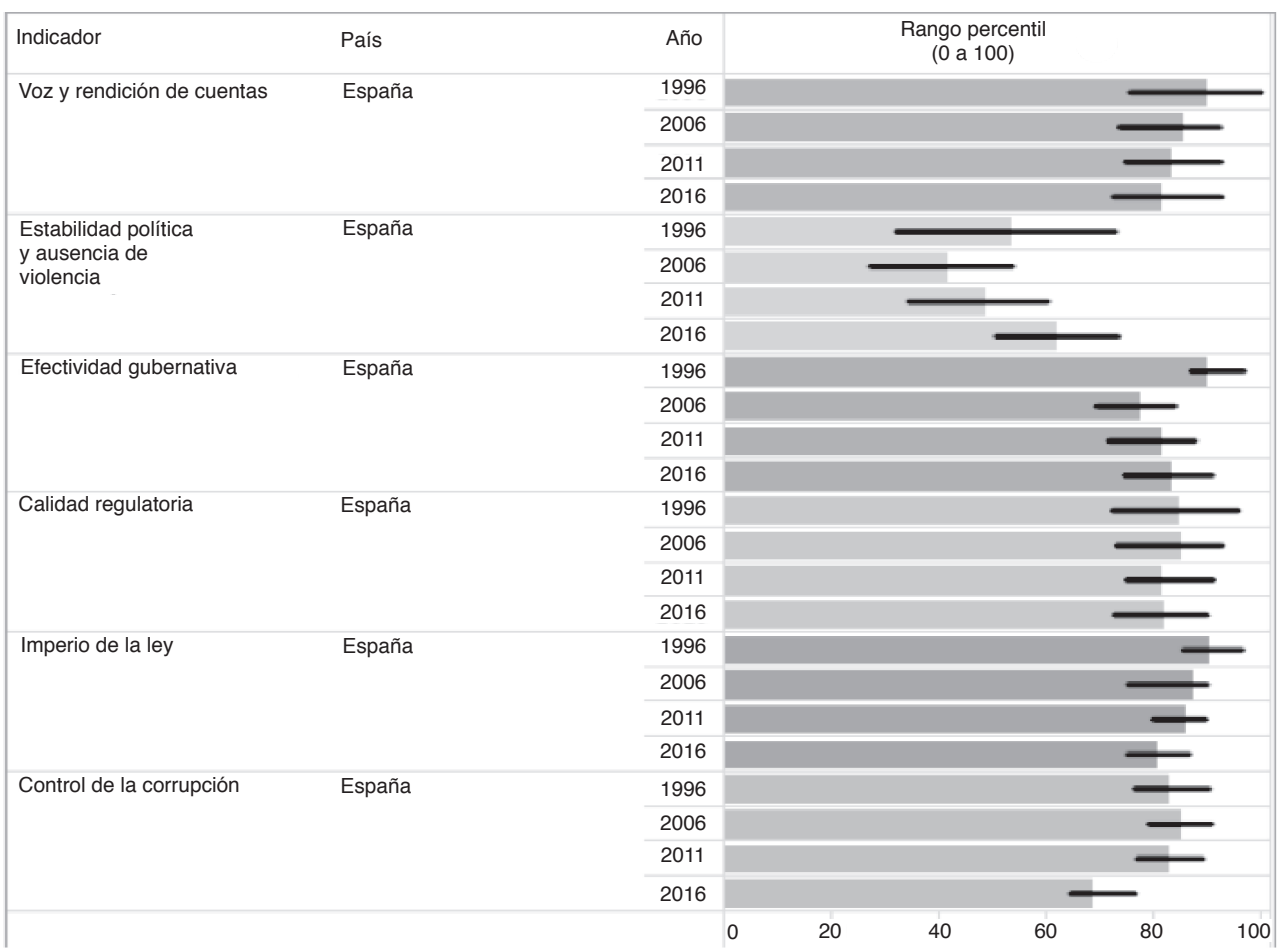

FUENTE: Worldwide Governance Indicators.

EE. UU. La fuente del crecimiento negativo de la productividad total de los factores parece haber sido el aumento de la mala asignación de los factores de producción entre los distintos sectores económicos, especialmente en industrias en las que la influencia del sector público es mayor (por ejemplo, mediante licencias o reglamentaciones). Estas industrias más cercanas al sector público experimentaron aumentos significativamente mayores en esa mala asignación (García-Santana et al., 2013).

\subsection{Buen gobierno}

Es necesario que la población crea en la imparcialidad de las administraciones para que el estado de bienestar se consolide. El capitalismo corrupto, de amiguetes e influencias, arruina esa confianza. Todos los países se enfrentan a un desafío enorme pero bien conocido: ¿Cómo conciliar el capitalismo, el gobierno de unos pocos, con la democracia, el gobierno de muchos? ¿Cómo funcionará el capitalismo democrático?

La democracia no es suficiente para construir un buen gobierno. Según Charron et al. (2015), los tres factores que parecen tener el mayor apoyo empírico para 
comprender las diferencias en la calidad de la gobernanza entre países son: 1) una gestión pública profesional con una separación estricta entre las carreras de políticos y funcionarios; 2) descentralización y autonomía en la gestión de los recursos humanos, y 3) transparencia, entendida como el acceso a la información pública (sin publicidad ni ocultando los malos resultados) y libertad de prensa.

El buen gobierno público debe garantizar una acción justa por medio de instituciones gubernamentales imparciales (Holmberg et al., 2012). Cuando los recursos públicos se malbaratan entre la corrupción y el amiguismo, en lugar de utilizar criterios objetivos (como la mejora en la calidad del aire o los puntajes de alfabetización y aritmética obtenidos por los estudiantes en diferentes escuelas), los ciudadanos se muestran más reacios a favorecer las políticas de bienestar con independencia de cuánto les beneficien.

Parece que la participación en los procesos de toma de decisiones sociales afecta al bienestar de las personas, y que afecta más la propia participación en el proceso que los resultados de este proceso: los inmigrantes sin derecho a voto en Suiza, por ejemplo, se benefician de los resultados, pero no de la participación en el proceso.

Los intentos de mejora de la calidad de gobierno conocen dos aproximaciones diferentes: la legal y la «económica».

1. Enfoque legal. Diseño de leyes, procedimientos, mecanismos de inspección y de penalización. Problemas:

- Demasiado énfasis en los controles exante que reducen una discrecionalidad conveniente si va acompañada de responsabilidad.

- Los mecanismos de inspección son costosos, lo que ocasiona una baja probabilidad de detección

- Lentitud de la justicia, que transmite sensación de impunidad.

- Las penalizaciones sociales resultan más efectivas que las derivadas de la aplicación de la ley, pero los factores culturales tienen inercia y no son fácilmente modificables.

- La corrupción es una trampa. Cuando hay mucha, la sociedad no la ve tan mal, lo que está en el origen, a su vez, de mucha corrupción, lo que constituye un círculo vicioso.

2. Enfoque económico. Incentivos, transparencia y competencia.

- Buena gestión pública. Exige un servicio funcionarial meritocrático con salarios adecuados y decisiones alejadas de los grupos de influencia.

- La transparencia (sobre financiación pública, resultados, etc.) requiere participación de la sociedad en la gestión gubernamental, libertad de información, prensa independiente, cultura democrática y participativa. 
- Con transparencia, la competencia es un arma potente para reducir la corrupción. Los gestores de las instituciones/empresas mal gestionadas (ineficientes o corruptas) son reemplazados, lo que genera incentivos exante a una buena administración.

Ha llegado el momento de introducir en España, con un horizonte de 30 años, la idea y práctica de la competencia por comparación en calidad (universidades, centros educativos, centros sanitarios, etc.) sin la necesidad de mercados. No habrá mejor gestión pública sin una mejor política, una mejor gobernanza, un concepto que incluya la revisión del financiamiento de los partidos políticos, la responsabilidad, la resolución de conflictos de intereses y la profesionalización e independencia de las funciones ejecutivas del sistema (Callejón et al., 2018).

Ciertamente, los valores sociales en España, basados en la encuesta de la Fundación $\mathrm{BBVA}^{8}$, respaldan las nociones de mérito o competencia mucho menos que en otros países europeos, y habrá que hacer algo para que España madure como sociedad. Es posible que se desee informar sobre los costos, el rendimiento y la calidad de los servicios financiados con fondos públicos y alentar la conciencia de los impuestos y las cotizaciones que se pagan (bastante ocultos hoy) para favorecer el interés de la ciudadanía por los asuntos colectivos.

\subsection{La mejora de la gestión pública en España}

España tiene un problema con su gestión pública. Será muy difícil mejorar la gestión pública o introducir reformas que aumenten significativamente nuestra productividad sin una mejora en la calidad de la política y las instituciones que la condicionan. Los requisitos para un mejor gobierno del Estado son tan conocidos como ignorados: proteger el financiamiento de los partidos políticos limitando los gastos y controlando las contribuciones privadas; racionalizar las regulaciones electorales para acercarse al cumplimiento del principio de «una persona, un voto»; e independencia de los medios de comunicación públicos. Solo un Estado efectivo que facilite el tipo de instituciones transparentes e imparciales de países como los escandinavos (o muchos países de Europa Central) permitirá que España se fortalezca después de la crisis. Los partidos políticos juegan un papel irremplazable en todos los sistemas democráticos. A diferencia de lo que sucede en otras democracias avanzadas, en España el marco legal vigente no facilita la depuración de los partidos, aunque esto se ha revelado durante mucho tiempo como necesario. Los partidos españoles son autorregulados: los congresos y los órganos rectores se reúnen cuando es aconsejable para sus líderes; el método usual para seleccionar posiciones internas y candidatos para puestos representativos es la cooptación; y los organismos de control están

${ }^{8}$ Fundación BBVA. Estudio internacional Values and Worldviews, 2013. http://www.observatorio religion.es/upload/39/47/Estudio_Fundacion_BBVA_sobre_estilos_de_vida_valores_y_creencias.pdf. 
fuertemente politizados. En las democracias constitucionalmente más avanzadas, los partidos están fuertemente regulados por la ley o, como en el caso británico, por la costumbre. En todos los países hay corrupción política, pero la democracia interna en los partidos políticos, la competencia entre aquellos que son líderes y aquellos que aspiran a serlo, y la obligación de transparencia impuesta por la ley permiten que los políticos corruptos sean rápidamente destituidos. En España esto no sucede, y la corrupción crece, debilita la acción gubernamental en una coyuntura crítica, provoca el descontento de los ciudadanos y termina provocando una grave crisis para la politización y la pérdida de eficiencia de las instituciones estatales. Hay aún más. El método de cooptación, repetido una y otra vez, es un método de selección adverso que acaba promoviendo a los menos críticos y menos capaces a los lugares de responsabilidad. Parece urgente desarrollar una nueva ley de partidos políticos, a fin de regular su actividad, garantizar su democracia interna, mejorar la transparencia y control de la financiación, y acercar la política a los ciudadanos. Esta es una condición necesaria para poder lanzar una reforma institucional mucho más amplia con garantías razonables que deberían incluir, entre otras, la reforma de la justicia, la regulación de los lobbies y la separación estricta de las posiciones políticas y administrativas para garantizar la independencia y el profesionalismo de la función pública. Las reglas básicas que debe reunir esta nueva ley son muy comunes en las democracias europeas.

Una mejora en la calidad de la política permitirá actuar sobre los factores que directamente contribuyen a la buena gobernanza y que se presentan como vulnerables al no resultar, como mínimo en apariencia, de una larga evolución histórica.

\section{Vías de avance en gestión sanitaria}

\subsection{Financiación pública}

Para que el acceso a los servicios sanitarios dependa de la necesidad clínica y sanitaria, la práctica totalidad de los países desarrollados del mundo optan por su financiación pública ${ }^{9}$ : aseguramiento universal y obligatorio contribuyendo según capacidad ante la inviabilidad de la solución coasiana -el aseguramiento voluntario de colectivos- por la concentración del gasto en colectivos deprimidos. Lo que parece manifestar unos valores de equidad tiene también una justificación en términos de eficiencia, dado que, si se quiere obtener la máxima cantidad y calidad de vida con los recursos disponibles, hay que dejar las extravagancias de lado para centrarse en lo clínicamente efectivo. Siempre cabrá satisfacer caprichos según la capacidad y voluntad de pagar individualmente por los mismos.

9 «An affordable necessity. Both in poor and rich countries, universal health care brings huge benefits». The Economist, 28 abril 2018. 
La salud de la población es tanto un instrumento como un indicador de desarrollo. Su financiación pública tendrá éxito en la medida en que se actúe sobre los determinantes sociales con una perspectiva de salud pública y huyendo de las rigideces que los grupos de interés, industriales, corporativos y profesionales introducen (Bloom et al., 2018). Por otra parte, en España la mayor financiación pública no ha de suponer revisión de tipos pero si eliminación de beneficios fiscales (IRPF e IVA), actuación en impuestos especiales, particularmente introduciéndolo sobre las bebidas azucaradas, y un mayor uso de precios públicos. Además, la armonía social y el individualismo encauzado aconsejan, como recomienda el World Inequality Report 2018, progresividad impositiva, registro global en el que conste la propiedad de los activos financieros (antídoto del lavado de capitales, la evasión fiscal y la creciente desigualdad), y mejor acceso a la educación (y a ocupaciones bien remuneradas).

\subsection{Hacer individualmente atractivo lo socialmente conveniente}

La conquista de la Humanidad llamada estado de bienestar puede consolidarse en España, incluida su joya de la corona, la atención sanitaria, con ligeros retoques, por un lado, que lo hagan más parecido a los estados de bienestar nórdicos y del centro de Europa y, por otro, adoptando medidas que la realidad y numerosos dictámenes reclaman. Entre otras:

- Recuperar la planificación (esa competencia para autorizar aberturas, modificaciones o cierres de instalación sanitaria), que al dimensionar la oferta humana y física condiciona en altísimo grado la utilización del futuro. Esto es, se precisa una auténtica gestión de la utilización.

- Hacer efectivo el factor de sostenibilidad que regule la composición de la cartera de servicios sanitaria según coste-efectividad e impacto presupuestario tal como hacen los países europeos con más poder adquisitivo (y con un estado de bienestar más consolidado).

La práctica clínica se adapta a la oferta disponible en cuanto a medios a disposición y cartera de servicios establecidos, como repetidas veces se ha mostrado desde la famosa «historia de dos ciudades»: Boston y New Haven. La práctica clínica, por otra parte, como principal asignadora de recursos sanitarios -en decisiones diagnósticas y terapéuticas-, contiene la clave para que un sistema sanitario financiado públicamente sea deseable para los ciudadanos votantes (Peiró, 2003): que sea solvente, que tenga capacidad resolutiva, lo que implica, con recursos limitados, tanto eliminar el exceso de la utilización inadecuada, de la sobreutilización y de la infrautilización -un tercio del gasto sanitario en EE. UU.- (Institute of Medicine, 2010), como reducir la brecha entre la eficacia (lo que idealmente podría conseguirse) y la efectividad (lo que realmente se está consiguiendo). 
Hacer individualmente atractivo para los clínicos una mejora continua de su práctica aconseja posibilitar la existencia de organizaciones con autonomía de gestión, que reciban una parte de sus presupuestos en función de resultados -ajustando por todo lo que hay que ajustar- en un entorno de competencia por comparación en calidad, para lo que se precisa una mejor política que permita una mejor gestión pública (Meneu y Ortún, 2014).

\section{Referencias bibliográficas}

[1] ARRUÑADA, B. (1994). «La estructura contractual de la empresa». En R. Febrero (ed.), Qué es la Economía. Madrid, Pirámide, 583-615.

[2] BLOOM, D.; KHOURY, A. y SUBBARAMAN, N. (2018). «The promise and peril of universal health care». Science 361, eaat9644. DOI: 10.1126/science.aat9644

[3] BLOOM, M.; PROPPER, C.; SEILER, S. y REENEN, J. (2015). «The impact of competition on management quality: evidence from public hospitals». Review of Economic Studies, 82, 457-489.

[4] BLOOM, N.; GENAKOS, C.; SADUN, R. y REENEN, J. (2012). «Management practices across firms and countries». NBER Working Paper No. 17850, Cambridge, Massachusetts.

[5] BRADLEY, E. H.; CURRY, L. A. y SPATZ, E. S. et al. (2012). «Hospital strategies for reducing risk-standarized mortality rates in acute myocardial infraction». Annals of Internal Medicine, 156 (9), 618-626.

[6] BRUGUÉS, A.; PERIS, A.; PAVÓN, F.; MATEO, E.; GASCÓN, J. y FLORES, G. (2016). «Evaluación de la gestión enfermera de la demanda en atención primaria». Atención Primaria, 48 (3), 159-165.

[7] CALLEJÓN, M.; CAMPILLO-ARTERO, C. y ORTÚN, V. (2018). «Deciding on public programs: prioritisation and governance». En J. del Llano y S Peiró (dirs.), Prioritising Health Services or Muddling Through. Madrid, Springer Healthcare, 1-22.

[8] CHANDLER, A. (1990). Scale and Scope. The Dynamics of Industrial Capitalism. Cambridge, Massachusetts, Harvard University Press.

[9] CHARRON, N.; DIJKSTRA, L. y LAPUENTE, V. (2015). «Mapping the Regional Divide in Europe: A measure for assessing quality of government in 206 European Regions». Soc. Indic. Res., 122, 315-346.

[10] CUTLER, D.M. y MCCLELLAN, M. (2001). «Is technological change in medicine worth it? Health Affairs (Milwood), 2001, 3. 11-29.

[11] ESTEBAN, F.; GARCÍA, M. y SANZ, R. et al. (2012). «Resultados de la implantación de un protocolo de fast-track en una unidad de cirugía colorrectal: estudio comparativo». Cirugía Española, 90, 434-9.

[12] FERNÁNDEZ-VILLAVERDE, J.; GARICANO, L. y SANTOS, T. (2013). «Political credit cycles. The case of the Eurozone». Journal of Economic Perspectives, 27 (3), $145-66$.

[13] FOGEL, R. (1994). «Economic growth, population theory and physiology: the bearing of long-term processes on the making of economic policy». American Economic Review, 84 (3), 369-395. 
[14] GARCÍA-ALTÉS, A.; SUELVES, J. M. y BARBERÍA, E. (2013). «Cost savings associated with 10 years of road safety policies in Catalonia, Spain». Bull. World Health Organ., 91 (1), 28-35.

[15] GARCÍA-ALTÉS, A.; PINILLA, J. y MARÍ-DELL'OLMO, M. et al. (2015). «Economic Impact of Smoke-Free Legislation: Did the Spanish Tobacco Control Law Affect the Economic Activity of Bars and Restaurants?». Nicotine Tob. Res., 17 (11), 1397-400.

[16] GARCÍA-ALTÉS, A.; COLLS, C.; ABILLEIRA, S. y GALLOFRÉ, M. (2016). «L'avaluació de polítiques públiques en l'àmbit sanitari: la millora de l'atenció a l'ictus a Catalunya com a exemple». Nota d'Economia, 102, 164-74.

[17] GARCÍA-SANTANA, M.; MORAL-BENITO, E.; PIJOAN-MAS, J. y RAMOS, R. (2013). «Growing like Spain: 1995-2007». Madrid, Banco de España, Working Paper No. 1609.

[18] GIL-EGEA, M. J.; MARTÍNEZ, M. A. y SÁNCHEZ, M. et al. (2008). «Rehabilitación multimodal en cirugía colorrectal electiva. Elaboración de una vía clínica y resultados iniciales». Cirugía Española, 84, 251-5.

[19] HERNÁNDEZ-AGUADO, I. (2018). «Diseño institucional y buen gobierno: avances y reformas pendientes». Cuadernos Económicos de ICE.

[20] HOLMBERG, S. y ROTHSTEIN, B. (2012). Good Government: The Relevance of Political Science. Cheltenham, Reino Unido, Edgar Elgar.

[21] INSTITUTE OF MEDICINE (2010). The Health Care Imperative: Lowering Costs and Improving Outcomes. Washington DC: National Academies Press.

[22] JANKE, K.; PROPPER, C. y SADUN, R. (2018). «The Impact of CEOs in the Public Sector: Evidence from the English NHS». Working Paper 18-075. Cambridge, Massachusetts: Harvard Business School.

[23] KAY, J. (1993). The foundations of corporate success. Oxford University Press.

[24] LÓPEZ RODÓ, L. (1958). «La reforma administrativa en España». Revista de Documentación Administrativa, 1, 5-14.

[25] MANCHÓN-WALSH, P.; ESPINÀS, J. A.; PRADES, J.; TORRENTS, A.; ALISTE, L. y POZUELO, A. et al. (2017). «Avaluació dels resultats de la concentració de la cirurgia oncològica digestiva d'alta especialització a Catalunya: una actualització en què s'inclou el periode 2014-2015». Monogràfics de la Central de Resultats, núm. 28. Barcelona: Agència de Qualitat i Avaluació Sanitàries de Catalunya. Departament de Salut. Generalitat de Catalunya. http://observatorisalut.gencat.cat/web/.content/minisite/ observatorisalut/ossc_central_resultats/informes/fitxers_estatics/MONOGRAFIC_28_ concentraciocirurgia_CdR.pdf.

[26] McCONNELL, K. J.; LINDROOTH, R. C.; WHOLEY, D. R.; MADDOX, T. M. y BLOOM, N. (2013). «Management practices and the quality of care in cardiac units». JAMA Intern. Med., 173 (8), 684-692.

[27] McCONNELL, K. J.; LINDROOTH, R. C.; WHOLEY, D. R.; MADDOX, T. M. y BLOOM, N. (2016). «Modern management practices and hospital admissions». Health Economics, 25 (4), 470-85.

[28] McKEOWN T. (1976). The modern rise of population. Nueva York, Academic Press.

[29] MENEU, R.; ORTÚN, V. y RODRÍGUEZ ARTALEJO, F. Innovaciones en gestión clínica y sanitaria. Barcelona, Masson.

[30] MENEU, R. y ORTÚN, V. (2014). «Del buen gobierno y nuestras instituciones». Economía y Salud. Boletín Informativo, 80. http://www.aes.es/boletines/news.php? $i d B=21 \& i d N=1312$. 
[31] MILGROM, P. y ROBERTS, J. (1992). Economics, Organizations and Management. Pearson.

[32] NOVOA, A. M.; PÉREZ, K. y SANTAMARIÑA-RUBIO, E. et al. (2010a). «Effectiveness of speed enforcement through fixed speed cameras: a time series study». Inj. Prev., $16(1), 12-6$.

[33] NOVOA, A. M.; PÉREZ, K.; SANTAMARIÑA-RUBIO, E. et al. (2010b). «Impact of the penalty points system on road traffic injuries in Spain: a time-series study». Am.J. Public Health, 100 (11), 2220-7.

[34] NOVOA, A. M.; PÉREZ, K. Y SANTAMARIÑA-RUBIO, E. et al. (2011). «Road safety in the political agenda: the impact on road traffic injuries». J. Epidemiol. Community Health, 65 (3), 218-25.

[35] PEIRÓ, S. «De la gestión de lo complementario a la atención integral de la atención a la salud: gestión de enfermedades e indicadores de actividad». En. V. Ortún (dir.), Gestión clínica y sanitaria: de la práctica diaria a la academia, ida y vuelta. Barcelona: Masson-Elsevier, 17-87.

[36] PINILLA, J. (2017). ¿Cómo legislar para promover la salud pública? Los casos del tabaco y los accidentes de tráfico. Observatorio Social de "la Caixa". https:// observatoriosociallacaixa.org/-/como-legislar-para-promover-la-salud-publica_ los-casos-del-tabaco-y-los-accidentes-de-trafico.

[37] PONS, J. M. V. (2010). «Innovación en el sector salud». Medicina Clínica, 135 (15), 697-699.

[38] TAFUNELL, X. y CARRERAS, A. (2010). Historia económica de la España contemporánea 1789-2009. Madrid, Ed. Crítica.

[39] TSAI, T.; JHA, A.; GAWANDE, A.; HUCKMAN, R.; BLOOM, N. y SADUN, R. (2015). «Hospital board and management practices are strongly related to hospital performance on clinical quality metrics». Health Affairs, 34 (8), 1304-1311.

[40] VIDAL-ALABALL, J.; ÁLAMO-JUNQUERA, D.; LÓPEZ-AGUILÁ, S. y GARCÍA-ALTÉS A. (2015). «Evaluación del impacto de la teledermatología en la disminución de la lista de espera en la comarca del Bages 2009-2012». Atención Primaria, 47 (5), 320-321. 